\title{
Recurrence and Recovery of COVID-19 in an Older Adult Patient with Multiple Comorbidities: A Case Report
}

\author{
Shannon Leung ${ }^{a}$ Nasiffa Hossain ${ }^{b}$ \\ aSchool of Medicine, Virginia Commonwealth University, Richmond, VA, USA; bivision of Geriatric Medicine, \\ Virginia Commonwealth University Health System, West Hospital, Richmond, VA, USA
}

\section{Keywords}

Coronavirus disease $\cdot$ Recurrence $\cdot$ Reinfection $\cdot$ Older adult · Asymptomatic

\begin{abstract}
The coronavirus disease 2019 (COVID-19) pandemic has become a worldwide public health crisis since December 2019. Reports of COVID-19 recurrences are uncommon but raise the question of whether patients infected with severe acute respiratory syndrome coronavirus 2 (SARS-CoV-2) will develop lasting immunity against or whether there are multiple viral strains that need to be considered. Although severe disease typically occurs in older individuals with comorbidities, this case report describes a patient in this demographic group who presented with COVID-19 recurrence and remained relatively asymptomatic throughout both disease courses. This case highlights how SARS-CoV-2 appears to affect some patients unpredictably, indicating that more research is needed to further understand its viral pathophysiology and disease outcomes.

(c) 2021 S. Karger AG, Basel
\end{abstract}

\section{Introduction}

Initially detected in December 2019, severe acute respiratory syndrome coronavirus 2 (SARS-CoV-2) has caused over 28 million cases and nearly 500,000 deaths in the United States, as of February 2021 [1]. Older patients are most likely to experience severe symptoms of coronavirus disease 2019 (COVID-19) once infected, given the increased likelihood of comorbidities in this age-group [2].

Highly debated in the medical community is whether prior infection with SARS-CoV-2 confers lifelong immunity against the virus. There is reported evidence that patients previously infected with COVID-19 are seronegative for SARS-CoV-2 antibodies within 3 months after initial diagnosis, which supports the idea that recurrence or reinfection with COVID-19 is possible [3,4]. This case report describes an older adult patient with multiple comorbidities to not only be diagnosed with a COVID-19 recurrence but also to remain unexpectedly asymptomatic throughout both infections. karger@karger.com

www.karger.com/ger

Karger ${ }^{\prime}=$
(C) 2021 S. Karger AG, Basel

(2021 S. Karger AG, Basel




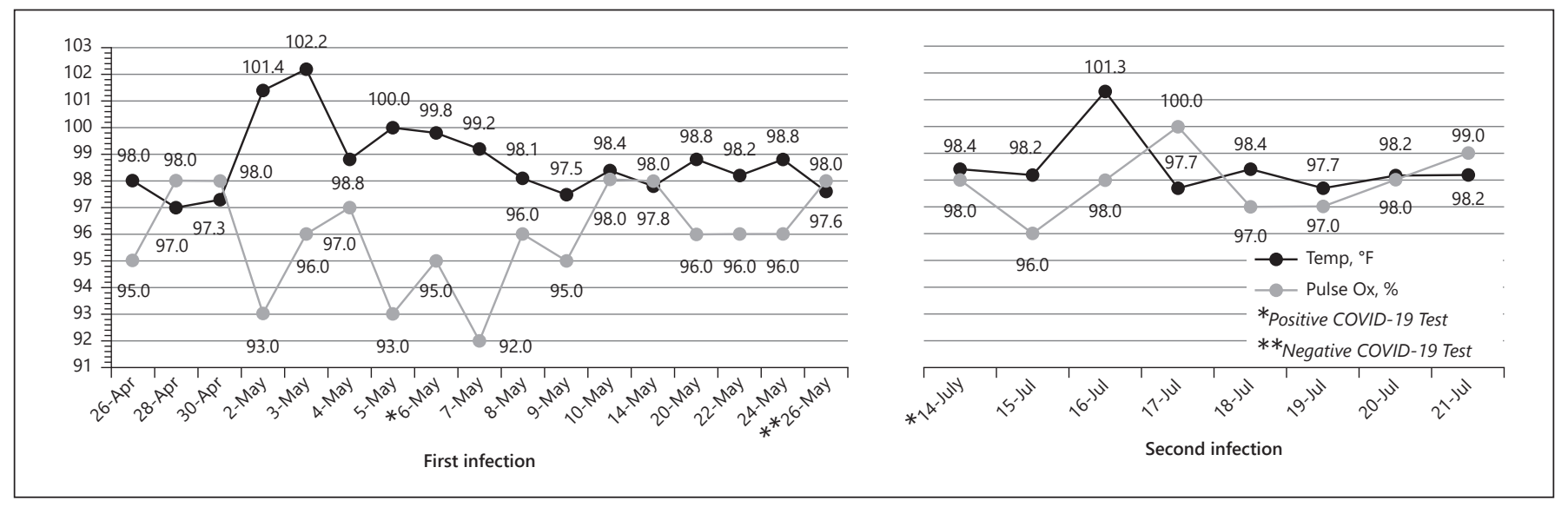

Fig. 1. Graphical representation of the patient's temperature (in black) and pulse oximetry (in gray) during the first and second COVID-19 infections. A positive COVID-19 test is denoted by a single asterisk $(*)$, and a negative COVID-19 test is denoted by a double asterisk $(* *)$. Patient was considered to be febrile at temperatures $>100.4^{\circ} \mathrm{F}\left(>38.0^{\circ} \mathrm{C}\right)$ and hypoxic at pulse oximetry $<95 \%$.

\section{Clinical Presentation}

A 69-year-old male from Virginia, United States, was discharged to a skilled nursing facility on 25 April 2020, after hospitalization for complications with a lumbar hardware infection. The patient had a history of multiple prior thoracolumbar fusions with recurrent Pseudomonas spine infection, spastic paraplegia with neurogenic bladder and bowel, prostate cancer status post radiation therapy, type 2 diabetes mellitus, hypertension, and hyperlipidemia. Over the next several days, a COVID-19 outbreak was reported at this skilled nursing facility. The patient developed an initial fever of $101.4^{\circ} \mathrm{F}\left(38.6^{\circ} \mathrm{C}\right)$ on 2 May 2020. The patient was given a RT-PCR test as part of protocol due to the outbreak. He was subsequently confirmed positive for COVID-19 on 6 May 2020.

Throughout his disease course, the patient developed a maximal fever of $102.2^{\circ} \mathrm{F}\left(39.0^{\circ} \mathrm{C}\right)$ on 3 May 2020 , and did not have any subsequent fevers (shown in Fig. 1). Pulse oximetry had the most variation between 2 May 2020, and 8 May 2020, with a low of $92 \%$ on 7 May 2020. Pulse oximetry stabilized after 9 May 2020 (shown in Fig. 1). Comprehensive metabolic panels, which include electrolytes, kidney function, and liver function tests, as well as complete blood counts remained mostly unremarkable except for 2 days of mild lymphopenia $\left(600 / \mathrm{mm}^{3}, 800 / \mathrm{mm}^{3}\right)$ and 1 day of mild neutrophilia $\left(8,200 / \mathrm{mm}^{3}\right)$. The patient remained otherwise asymptomatic.

The patient tested negative for COVID-19 using RTPCR on 26 May 2020. He was later discharged home from the skilled nursing facility on 8 June 2020 . The patient again tested negative for COVID-19 on 10 June 2020, which was obtained as routine precautionary testing prior to a surgical procedure that was unrelated to COVID-19. He remained out in the community after this procedure.

Patient had an episode of syncope during an outpatient appointment on 14 July 2020 and was transported to the emergency department for hospital admission. He was tested for COVID-19 via RT-PCR testing as part of routine preadmission screening, in which he was found to be positive.

The patient did not develop fever until 2 days after his episode of syncope. He had a maximal temperature of $101.3^{\circ} \mathrm{F}\left(38.5^{\circ} \mathrm{C}\right)$ on 16 July 2020 but had no fevers on subsequent days (shown in Fig. 1). Pulse oximetry ranged from $96 \%$ to $100 \%$ throughout his hospital stay between 14 July 2020 and 21 July 2020 (shown in Fig. 1). Chest xray revealed no acute changes. Extensive cardiac and infectious workup was negative, and etiology for patient's syncope was determined to be unrelated to COVID-19 and likely vasovagal in nature. Patient was eventually discharged from the hospital on 21 July 2020.

\section{Discussion}

Recurrence of COVID-19 has been well documented in case reports, but the nature of the recurrence is still debated [5]. Scientists have argued whether recurrence is due to reactivation of latent strains or infection with a dif- 
ferent strain of SARS-CoV-2. Regardless of the cause, these documented recurrences of SARS-CoV-2 have important implications on the global management of the COVID-19 pandemic.

Immunosuppression from drugs or pathological conditions may result in suboptimal control of SARS-CoV-2, leading to viral reactivation [6]. Our patient was not on chemotherapy or corticosteroids at the time of either COVID-19 diagnoses; however, he did have multiple comorbidities that placed him at higher risk of developing COVID-19, which included recently acquired spastic paraplegia, recurrent lumbar hardware infections requiring surgical interventions, diet-controlled type 2 diabetes mellitus, and hypertension. It is likely our patient first acquired SARS-CoV-2 through the outbreak at his skilled nursing facility. Despite making a seemingly full recovery with two subsequent negative COVID-19 tests, the patient reacquired the virus 2 months later. It is possible that the second COVID-19 infection was due to reinfection of the same SARS-CoV-2 strain because of suboptimal initial immune response from his multiple immunosuppressive factors [6].

The other possibility is that our patient's two positive COVID-19 tests were a result of a single infection with prolonged viral clearance rather than a second infection. It has been documented that viral shedding can be detected for as long as 60 days after onset of symptoms [7]. This timeline is close to that of our patient's, with 72 days between his initial onset of symptoms on 2 May 2020 and his second COVID-19 positive test on 14 July 2020. However, it should be highlighted that the patient had two consecutive negative COVID-19 tests in between his two positive results and he remained asymptomatic except for $1-2$ days of fever $\left(>100.4^{\circ} \mathrm{F},>38.0^{\circ} \mathrm{C}\right)$ throughout his infections, which suggests that the explanation of a prolonged single infection is unlikely.

At least 54 global viral strains of SARS-CoV-2 have already been documented, and possible infection with a mutated SARS-CoV-2 strain must also be considered. With whole genome sequencing, there has been at least one report of COVID-19 recurrence with a genetically distinct SARS-CoV-2 strain from that of the first infection [8]. It should be noted that the patient described in this case report was discharged from his skilled nursing facility in between his two positive COVID-19, which would make reinfection of the same SARS-CoV-2 strain less likely and infection with a novel strain more likely. Unfortunately, our patient's pharyngeal samples were discarded shortly after testing and we were unable to investigate further.

COVID-19 Recurrence in Older Adult Patient
Asymptomatic or mildly symptomatic patients with COVID-19 have mainly been reported in younger and middle-aged individuals without underlying diseases [9]. However, the older adult patient with multiple comorbidities as described in this case report remained only mildly symptomatic during both COVID-19 infections with $1-2$ days of fever $\left(>100.4^{\circ} \mathrm{F},>38.0^{\circ} \mathrm{C}\right)$, largely normal pulse oximetry $(>95 \%)$, and unremarkable chest $x$-ray. In addition, the patient's positive COVID-19 tests were incidental findings - the first infection due to mass testing at his skilled nursing facility, and the second due to mandated hospital preadmission testing for symptoms unrelated to COVID-19. This raises the question of whether our patient was a unique case or perhaps the more likely scenario of whether this is a common undocumented occurrence, which would suggest that there may be an even higher number of COVID-19 cases and lower case-fatality rate than currently reported.

Lastly, inaccurate testing could explain the COVID-19 recurrence seen in this case report. It is possible that our patient had a single prolonged single infection, testing falsely negative twice in between his two COVID-19 positive tests. Because the false negative rate of the RT-PCR test is reported to be between $8 \%$ and $18 \%$ [10], it is possible for our patient to be having a COVID-19 infection despite testing falsely negative two consecutive times. Unfortunately, our patient did not undergo any COVID-19 antibody testing. A positive antibody test may have been helpful to distinguish a single prolonged infection with a low viral load, which could explain the falsely negative RT-PCR results. Another possible explanation would be that the patient had a false positive test on 14 July 2020, since he had no known exposures at the time, unlike his first positive test on 6 May 2020, in which he was exposed during a COVID-19 outbreak at his skilled nursing facility. Although possible, it is still unlikely given that the false positive rate for the RT-PCR test is between $1 \%$ and $2 \%[10]$.

In conclusion, this case report describes an uncommon presentation of a patient with COVID-19 recurrence. Although rare, reports of these recurrences are important to help guide future research on understanding the immunology of SARS-CoV-2 and potential treatments to overcome the COVID-19 pandemic.

\section{Statement of Ethics}

Written informed consent was obtained from the patient for publication of this case report and any accompanying images.

Gerontology 2021;67:445-448

DOI: $10.1159 / 000514675$ 


\section{Conflict of Interest Statement}

The authors have no conflicts of interest to declare.

\section{Funding Sources}

This research did not receive any specific grant from funding agencies in the public, commercial, or not-for-profit sectors.

\section{Author Contributions}

Leung, S. was responsible for literature search, writing, drafting, and revising, final approval, and agrees to be accountable for all aspects of the manuscript. Hossain, N. was responsible for literature search, drafting, and revising, final approval, and agrees to be accountable for all aspects of the manuscript.

\section{References}

1 CDC.https://www.cdc.gov/covid-data-tracker.

2 Liu K, Chen Y, Lin R, Han K. Clinical features of COVID-19 in elderly patients: a comparison with young and middle-aged patients. J Infect. 2020 Jun $1 ; 80(6)$ :e14-8.

3 Liu A, Wang W, Zhao X, Zhou X, Yang D, Lu $\mathrm{M}$, et al. Disappearance of antibodies to SARS-CoV-2 in a -COVID-19 patient after recovery. Clin Microbiol Infect. 2020 Dec; 26(12):1703-5. Available from: http://www. sciencedirect.com/science/article/pii/ S1198743X20304110.

4 Long QX, Tang XJ, Shi QL, Li Q, Deng HJ, Yuan J, et al. Clinical and immunological assessment of asymptomatic SARS-CoV-2 infections. Nat Med. 2020 Aug;26(8):1200-4.

5 Lafaie L, Célarier T, Goethals L, Pozzetto B, Grange S, Ojardias E, et al. Recurrence or relapse of COVID-19 in older patients: a description of three cases. J Am Geriatr Soc. 2020 Oct;68(10):2179-83. Available from: http://onlinelibrary.wiley.com/doi/abs/ 10.1111/jgs.16728.

6 Gousseff M, Penot P, Gallay L, Batisse D, Benech N, Bouiller K, et al. Clinical recurrences of COVID-19 symptoms after recovery: viral relapse, reinfection or inflammatory rebound? J Infect. 2020 Nov; 81(5): 816-46. Available from: http://www. sciencedirect.com/science/article/pii/ S0163445320304540.

7 Li J, Zhang L, Liu B, Song D. Case report: viral shedding for 60 days in a woman with novel coronavirus disease (COVID-19). Am J Trop Med Hyg. 2020 Jun;102(6):1210-3. Available from: https://www.ncbi.nlm.nih.gov/pmc/ articles/PMC7253087/.
8 To KK-W, Hung IF-N, Ip JD, Chu AW-H, Chan W-M, Tam AR, et al. COVID-19 re-infection by a phylogenetically distinct SARScoronavirus- 2 strain confirmed by whole genome sequencing. Clin Infect Dis. 2020 Aug 25:ciaa1275. Available from:

9 Wang Y, Liu Y, Liu L, Wang X, Luo N, Li L. Clinical outcomes in 55 patients with severe acute respiratory syndrome coronavirus 2 who were asymptomatic at hospital admission in Shenzhen, China. J Infect Dis. 2020 May; 221(11):1170-4. Available from: https://www. ncbi.nlm.nih.gov/pmc/articles/PMC7184401/.

10 Baron RC, Risch L, Weber M, Thiel S, Grossmann K, Wohlwend N, et al. Frequency of serological non-responders and false-negative RT-PCR results in SARS-CoV-2 testing: a population-based study. Clin Chem Lab Med. 2020 Aug 31;58(12):2131-40..; 